\title{
Comparison Between Classification Using Impact Parameter and Using Number of Participants in Relativistic Nuclear Collisions
}

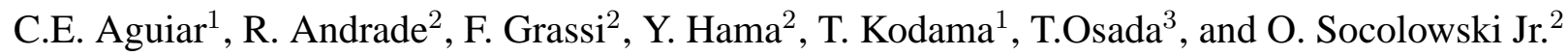 \\ ${ }^{1}$ Instituto de Física, Universidade Federal do Rio de Janeiro, C. P. 68528, 21945-970 Rio de Janeiro-RJ, Brazil \\ ${ }^{2}$ Instituto de Física, Universidade de São Paulo, C. P. 66318, 05315-970 São Paulo-SP, Brazil \\ ${ }^{3}$ Universidade Ochnomizu, Tóquio, Japão
}

Received on 15 August, 2003.

\begin{abstract}
Using the hydrodynamical code NeXSPheRIO, we compare predictions as usually done in hydrodynamics, using centrality windows defined through the impact parameter, and as obtainable experimentally, using windows in participant number.
\end{abstract}

\section{Introduction}

Relativistic heavy-ion collisions as performed at the AGS, SPS and RHIC, can be classified as central (nuclei overlap), peripheral (nuclei pass grazing each other) or semiperipherical. Quantitatively, one can classify these collisions using the impact parameter $b$ : zero for central collisions, sum of the radii for peripheral, etc. More precisely, one can define a centrality window as incorporating the $n \%$ most central collisions i.e. all $b$ 's solution of $\pi b^{2} / \sigma_{\text {inel }}=n$ $\%)$. This criteria to define centrality window is purely geometrical.

When the impact parameter is chosen, one can determine the initial conditions and run a hydrodynamical code to obtain predictions for such quantities as transverse momentum and rapidity spectrum, particle abundances, elliptic flow, etc, for various windows in impact parameter.

Experimentally, however, one does not have access to direct measurement of the impact parameter. So experimental results are presented in windows of energy deposited in a zero-degree calorimeter, number of participants, multiplicity, etc. One expects that the $n \%$ most central collisions in term of impact parameter are also the $n \%$ collisions with lower energy in the zero-degree calorimeter, higher number of participants and higher multiplicity.

However fluctuations are expected, for example the same value for the impact parameter may lead to somewhat different values of the number of participants due to the probabilistic nature of nucleon-nucleon collisions. The aim of this paper is to compare predictions using a classification of events using the impact parameter, the standard approach in hydrodynamics, and using the number of participants, as obtained experimentally. We also confront these two approaches to experimental data obtained at SPS by the NA49 collaboration, to turn the comparison more realistic.

All predictions are made using the NexSPheRIO code. The initial conditions are given by the NeXus code[1] for nuclear collisions and so fluctuate from event to event. These initial conditions are then used as input for SPheRIO, a 3+1 hydrodynamical code[2] based on a technique called Smoothed Particle Hydrodynamics.

\section{Determination of the centrality windows}

Using NeXus, $800 \mathrm{~Pb}+\mathrm{Pb}$ collisions at beam energy of 158 GeV A were simulated. In Fig. 1a, we show the resulting distribution of participating nucleons. Events were binned in six classes that contain 5\%, 9\%, 9\%, 8\%, $17 \%$ and $52 \%$ of the total number of collisions (going from the most central to peripheral), respectively. We explain later why we made this particular choice of classes.

As indicated in the previous section, a fixed value of $b$ can lead to various values of $N_{\text {part }}$ and vice versa. In Fig. $1 \mathrm{~b}$, we show for each of our 800 events, its precise value of $b$ and $N_{\text {part }}$. The horizontal lines are the windows in term of $N_{\text {part }}$ as defined above and in Fig. 1a. The vertical lines are the windows in term of the impact parameter. They are defined by solving in term of $b$ the equation $\pi b^{2} / \sigma_{\text {inel }}=$ $0-5 \%, 5-14 \%, 14-23 \%, 23-31 \%, 31-48 \%, 48-100 \%$ with $\sigma_{\text {inel }}=\pi(2 R)^{2}$ and $R$ the nuclear radius (this neglects boundary effects). In Fig. 1b, we see that the rectangles delimited by a continuous line include events that belong to a same window, independently of the classification used, $N_{\text {part }}$ or $b$. However if we used a $N_{\text {part }}$ classification for e.g. window 1 , we add to the rectangle $(1,1)$, the events in the $(2,1)$ rectangle while if use the $b$ classification, we would add instead the events in the $(1,2)$ rectangle. In this case, the number of events in $(1,2)$ or $(2,1)$ is small. However if we do the same for rectangle $(3,3)$, this is no more the case. As a consequence, the average number of participants is modified when going from one classification to the other. The average 

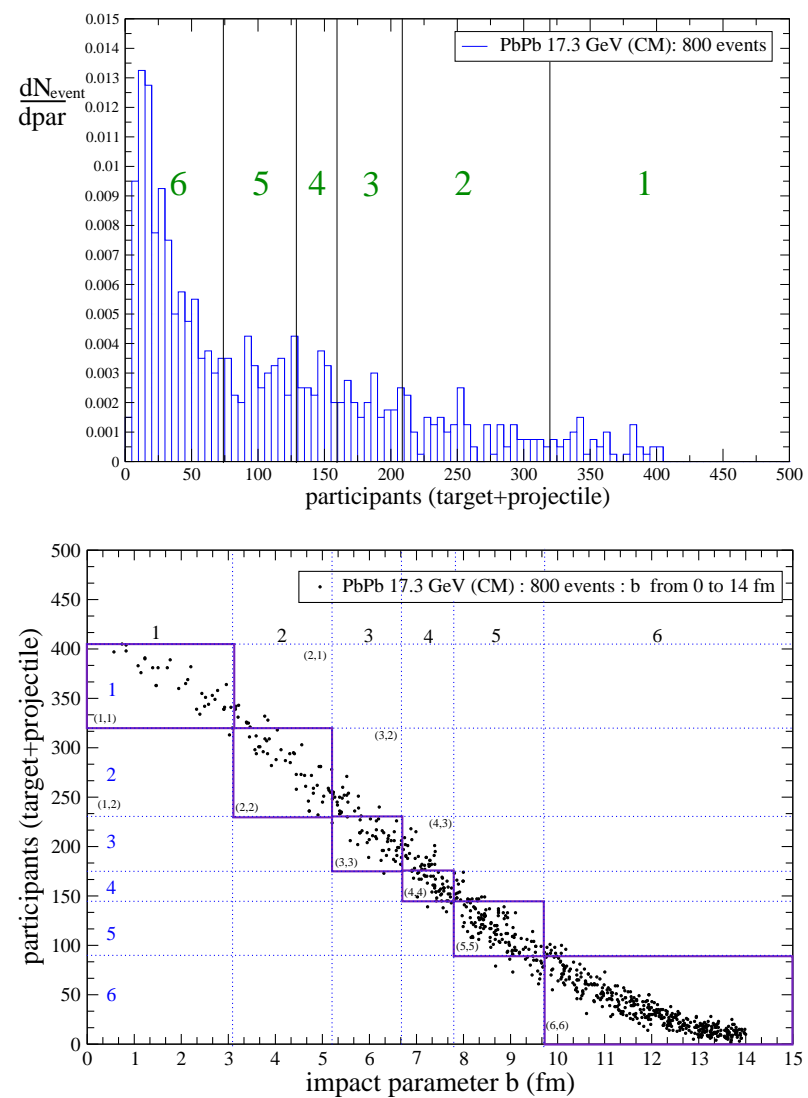

Figure 1. Results obtained with NeXus: a) (left) distribution of participant number and resulting centrality windows (see text) b) (right) relation between participant number and impact parameter (each point corresponds to one collision). Horizontal lines limit windows in $N_{\text {part }}$ while vertical lines limit windows in $b$.

number of participants for each window and each classification is shown in the table below. In the next section, we explore consequences of this.

We now explain why we made our particular choice of centrality windows. To classify our collisions in a realistic way, we use data obtained by the NA49 collaboration, shown in Fig. 2. In this case, the amount of energy deposited in a zero-degree calorimeter was used to select events with a given centrality[3].

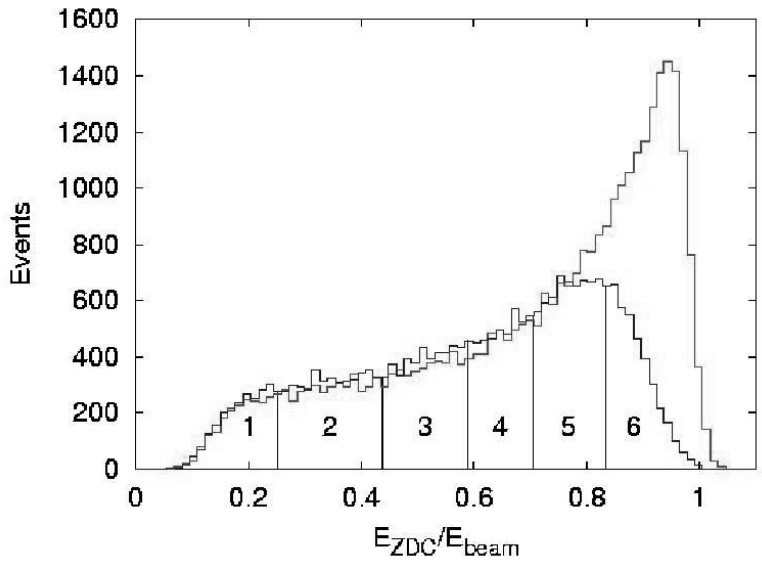

Figure 2. Results obtained by NA49 for the distribution of energy in the zero-degree calorimeter (solid line: with interaction trigger, dashed line: unbiased values) and their centrality windows.

In an ideal situation, we expect that spectator nucleons free-stream towards the zero-degree calorimeter and deposit their energy there. Then the number of spectators would be the ratio between the deposited energy $E_{Z D C}$ by the beam energy for the nucleon-nucleon collision $(158 \mathrm{GeV}$ in our case) and the number of participating nucleons would $N_{\text {part }}=A-E_{Z D C} / 158$. In practice, this is an approximation because there may be other collisions than $\mathrm{Pb}+\mathrm{Pb}$ depositing energy (such as $\mathrm{Pb}+$ air occurring because the zerodegree calorimeter is very far from the target and which can be corrected for to a certain extent), the beam energy fluctuates, etc. However as a guideline, we may consider that the $5 \%$ collisions with lower $E_{Z D C}$ are also the $5 \%$ collisions with higher number of participants, and so on. With this assumption, the NA49 classification which bins the events in Fig. 2 in six classes that contain 5\%, 9\%, 9\%, 8\%, 17\% and $52 \%$ of the total distribution of $E_{Z D C}$ would be the same as our classification of events in six classes of $N_{\text {part }}$. In the table below, for each NA49 window in $E_{Z D C}$, the mean number of participants is shown. This number of participating nucleons was estimated by NA49 in a way independent of the $E_{Z D C}$ measurement, by integrating rapidity distributions. As a result, it may be wiser not to compare the $<N_{\text {part }}>$ of our two classifications, with that of NA49.

\begin{tabular}{||c|c|c||c|c||c|c||}
\hline \multicolumn{3}{||c||}{ NA49 classification } & \multicolumn{2}{c||}{$N_{\text {part }}$ classification } & \multicolumn{2}{c||}{$b$ classification } \\
\hline window & centrality & $\left\langle N_{\text {part }}\right\rangle$ & $N_{\text {part }}$ range & $\left\langle N_{\text {part }}\right\rangle$ & $b$ range in fm & $\left\langle N_{\text {part }}\right\rangle$ \\
1 & $0-5 \%$ & $362 \pm 12$ & $320-416$ & 358 & $0-3.1$ & 364 \\
2 & $5-14 \%$ & $304 \pm 16$ & $230-320$ & 268 & $3.1-5.2$ & 289 \\
3 & $14-23 \%$ & $241 \pm 16$ & $175-230$ & 200 & $5.2-6.7$ & 220 \\
4 & $23-31 \%$ & $188 \pm 16$ & $145-175$ & 158 & $6.7-7.8$ & 171 \\
5 & $31-48 \%$ & $130 \pm 14$ & $90-145$ & 116 & $7.8-9.7$ & 117 \\
6 & $48-100 \%$ & $72 \pm 8$ & $0-90$ & 35 & $9.7-14$ & 35 \\
\hline
\end{tabular}

\section{Particle distributions}

The number and distribution of particles in a window depend on the average number of participants, therefore they depend on the classification used. To quantify this, we run the hydrodynamical code $\mathrm{NeXSPheRIO}$ for $180 \mathrm{~Pb}+\mathrm{Pb}$ collisions at $153 \mathrm{GeV} \mathrm{A}$ and computed the rapidity and transverse momentum distribution for $p-\bar{p}$ and charged pions 

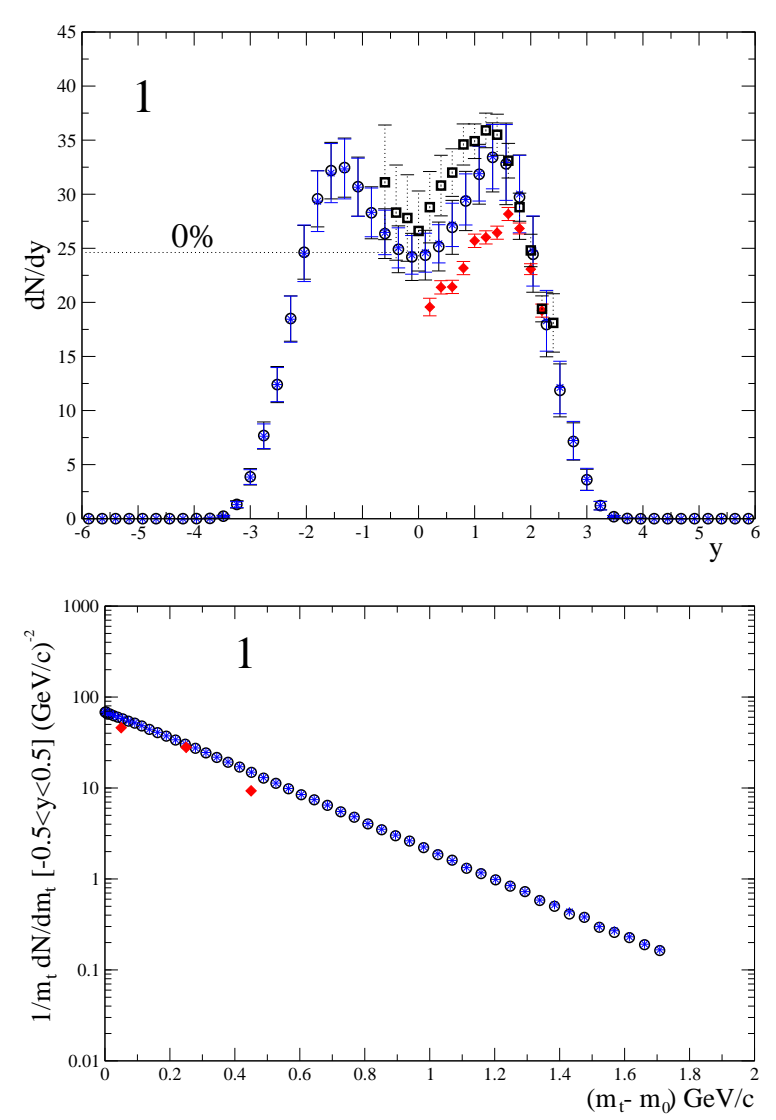

Figure 3. Results obtained with NeXSPheRIO for rapidity distribution of $p-\bar{p}$ and transverse mass distribution, both for window 1. Open circles are for impact parameter classification: $b$ from 0 to $3.1 \mathrm{fm}$ and stars are for participant number classification: $<N_{\text {part }}$ from 320 to 416 . No difference is seen between predictions from these two classifications in this window. For comparison, open squares are published data from NA49[4] and diamonds are data from a more recent unpublished reference[5].

in the various windows for our two classifications.

As explained above, in window 1, we do not expect large differences using one classification or another. This can indeed be checked in Fig. 3 for $p-\bar{p}$. However in window 3, we expect larger differences and this is shown in Fig. 4 for charged pions.

In our code, the initial conditions are fixed running NeXus, but we still have the freedom to adjust the freeze out temperature. In Figs. 3 and 4, we made the canonical choice $T_{\text {f.out }}=140 \mathrm{MeV}$, i.e. we did not try to adjust $T_{\text {f.out }}$ to best fit the data. However, we see that the hydrodynamical results are reasonably close to the data.

\section{Conclusion}

In this paper, we compared predictions as usually done in hydrodynamics, using centrality windows defined through the impact parameter, and as obtainable experimentally, using windows in participant number. We computed rapidity and transverse mass distributions for $p-\bar{p}$ and charged pions in various windows, and found no significant difference between the two classifications. This result corroborates those of Broniowski and Florkowski[6]. We feel however that a
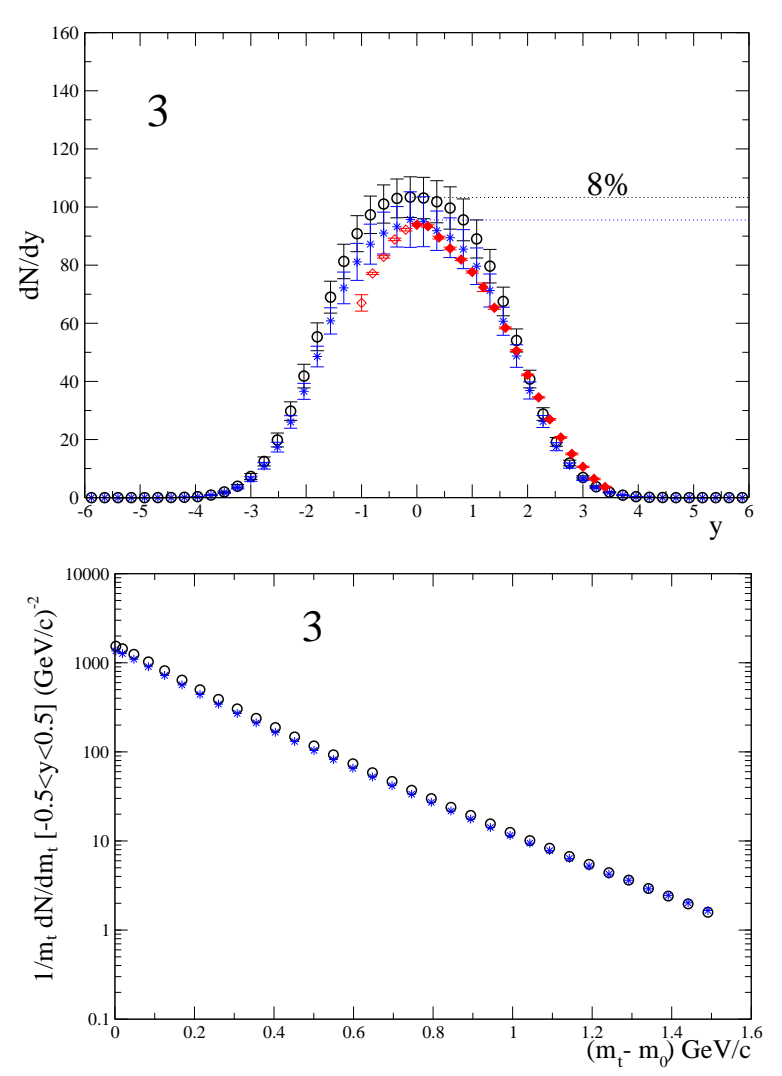

Figure 4. Results obtained with NeXSPheRIO for rapidity distribution of charged pions $/ 2$ and transverse mass distribution, both for window 3. Open circles are for impact parameter classification: $b$ from 5.2 to $6.7 \mathrm{fm}$ and stars are for participant number classification: $<N_{\text {part }}$ from 175 to 230 . An $8 \%$ difference at mid-rapidity is seen between predictions from these two classifications in this window. For comparison, diamonds are data from an unpublished reference[5].

more detailed study should be done for other quantities such as the elliptic flow and for other experimental classification, for example using multiplicity.

\section{Acknowledgements}

This work was partially supported by CAPES, CNPq, FAPERJ and FAPESP (2000/04422-7, 2000/05769-0, 2001/09861-1).

\section{References}

[1] H.J. Drescher, M. Hladik, S. Ostapchenko, T. Pierog, and K. Werner, Phys. Rep. 350, 93 (2001); H.J. Drescher, F.M. Liu, S. Ostapchenko, T. Pierog, and K. Werner, Phys. Rev. C 65, 054902 (2002).

[2] C.E. Aguiar, T. Kodama, T. Osada, and Y. Hama, J. Phys. G 27, 75 (2001); Nucl. Phys. A698, 639c (2002); Proceedings of the 6th International Workshop on RANP, World Scientific Ed., 2000.

[3] J. Bächler et al., Nucl. Phys. A661, 45c (1999).

[4] H. Appelshäuser et al., Phys. Rev. Lett. 82, 2471 (1999).

[5] G.E. Cooper, Ph. D. thesis, LBNL-45467 (2000).

[6] W. Broniowski and W. Florkowski, Phys. Rev. C 65, 024905 (2002). 